\title{
Effect of thermal radiation on free convection boundary layer flow over a vertical wavy cone
}

\author{
${ }^{a}$ E. M. A. Elbashbeshy, ${ }^{b}$ T.G. Emam, ${ }^{\text {c E. A. Sayed }}$ \\ ${ }^{a}$ Mathematics Department, Faculty of science, Ain Shams University, Abbassia, Cairo, Egypt, \\ b Mathematics Department, The German University in Cairo-GUC, New Cairo City, Cairo, Egypt, \\ ${ }^{c}$ Department of Physics\& Engineering Mathematics, Faculty of Engineering-Mattaria, Helwan University, \\ Cairo, Egypt,
}

\begin{abstract}
Effect of thermal radiation on a steady two-dimensional free convection laminar boundary layer flow of a viscous incompressible optically thick fluid over a vertical wavy cone has been investigated. Using appropriate transformations, the basic governing equations are transformed into non-dimensional boundarylayer equations. These equations are then solved numerically by using Mathematica technique. The effect of the radiation parameter on velocity, temperature, skin friction and local Nusselt number has been discussed with graphical representation.
\end{abstract}

Keywords: Laminar boundary layer, free convection, Vertical wavy cone, radiation effect.

\section{Introduction}

Convection problems associated with irregular surfaces have received less attention than the cases with regular surfaces. Surfaces are sometimes roughened to disturb the flow and alter the rate of heat transfer on such surfaces. Thus, it is clear that convection problems associated with wavy surfaces occur frequently in practice.

Many articles have been published [1-12] dealing the problem of natural convection over a vertical wavy cone, frustum of a wavy cone and wavy vertical surface.

Convective heat transfer with thermal radiation is great importance in processes involving high temperatures such as in gas turbines, nuclear power plants, and thermal energy storage among others. The thermal radiation of gray fluid which is emitting and absorbing in a nonscattering medium has been examined by Hossain et al. [13\&14], Raptis et al. [15], and Cortell [16-19].

In this article, the free convection boundary layer flow over a vertical wavy cone including thermal radiation effect has been investigated.

\section{Mathematical formulation}

The boundary layer analysis outlined below allows the shape of the wavy surface, $\hat{\sigma}(\hat{x})$ to be arbitrary, but our detailed numerical work will assume that the surface exhibits sinusoidal deformations. Thus the wavy surface of the cone is described by

$$
\hat{y}=\hat{\sigma}(\hat{x})=\hat{a} \sin (\pi \hat{x} / l)
$$

where $l$ is the fundamental wavelength associated with wavy surface and $\hat{a}$ is the amplitude of the surface waves.

The physical model of the problem and the two dimensional coordinate system are shown in Figure 1, where $\Omega$ is the half angle of the flat surface of the cone and $\hat{r}(\hat{x})$ is the local radius of the flat surface of the cone which is defined by

$\hat{r}=\hat{x} \sin (\Omega)$ 


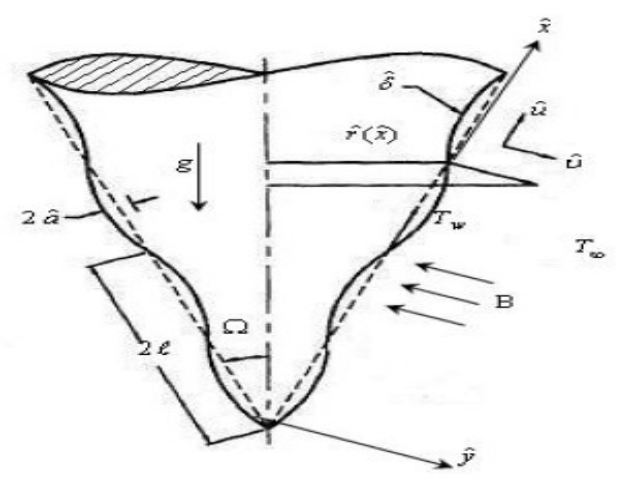

Fig. 1: Physical model and coordinates system

The conservation equations for the flow characterized with steady, laminar and two-dimensional boundary layer; under the usual Boussinesq approximation, the continuity, momentum and energy equations can be written as:

$$
\begin{aligned}
& \frac{\partial(\hat{r} \hat{u})}{\partial \hat{x}}+\frac{\partial(\hat{r} \hat{v})}{\partial \hat{y}}=0 \\
& \hat{u} \frac{\partial \hat{u}}{\partial \hat{x}}+\hat{v} \frac{\partial \hat{u}}{\partial \hat{y}}=-\frac{1}{\rho} \frac{\partial \hat{p}}{\partial \hat{x}}+v \hat{\nabla}^{2} \hat{u}+g \beta \cos \Omega\left(T-T_{\infty}\right) \\
& \hat{u} \frac{\partial \hat{u}}{\partial \hat{x}}+\hat{v} \frac{\partial \hat{u}}{\partial \hat{y}}=-\frac{1}{\rho} \frac{\partial \hat{p}}{\partial \hat{y}}+v \hat{\nabla}^{2} \hat{v}+g \beta \sin \Omega\left(T-T_{\infty}\right) \\
& \hat{u} \frac{\partial T}{\partial \hat{x}}+\hat{v} \frac{\partial T}{\partial \hat{y}}=\frac{k}{\rho C_{p}} \hat{\nabla}^{2} T-\frac{1}{\rho C_{p}} \frac{\partial q_{r}}{\partial \hat{y}}
\end{aligned}
$$

where $(\hat{x}, \hat{y})$ are the dimensional coordinates in the vertical and horizontal directions, $(\hat{u}, \hat{v})$ are the velocity components parallel to $(\hat{x}, \hat{y}), g$ is the acceleration due to gravity, $\hat{p}$ is the pressure of the fluid, $\rho$ is the density, $v$ is the kinematic viscosity, $\beta$ is the coefficient of volume expansion, $T$ is the temperature of the fluid, $T_{\infty}$ is the ambient temperature, $k$ is the thermal conductivity of the fluid, $C_{p}$ is the specific heat at constant pressure and $q_{r}$ is the radiative heat flux in the $\hat{y}$ direction.

The boundary conditions are given by

$\hat{u}=0, \hat{v}=0, T=T_{w}$ at $y=\hat{y}_{w}=\sigma(\hat{x})$

$\hat{u}=0, T=T_{\infty}$ as $\hat{y} \rightarrow \infty$

where $T_{w}$ is the surface temperature

Using the Rosseland approximation for radiation [15], radiative heat flux is simplified as

$q_{r}=-\frac{4 \sigma^{*}}{3 \alpha^{*}} \frac{\partial T^{4}}{\partial \hat{y}}$,

where $\sigma^{*}$ and $\alpha^{*}$ are the Stefan-Boltzmann constant and the Rosseland coefficient.

We assume that the temperature differences within the flow are such that the term $T^{4}$ may be expressed as a linear function of temperature. Hence, expanding $T^{4}$ in a Taylor series about $T_{\infty}$ and neglecting higher-order terms we get

$T^{4} \cong 4 T_{\infty}^{3} T-3 T_{\infty}^{4}$.

Using equations (8) and (9), the energy equation (6) becomes 
$u \frac{\partial T}{\partial x}+v \frac{\partial T}{\partial y}=\alpha \hat{\nabla}^{2} T+\frac{16 \sigma^{*} T_{\infty}^{3}}{3 \alpha^{*} \rho c_{p}} \frac{\partial^{2} T}{\partial \hat{y}^{2}}$

Now introduce the following non-dimensional variables

$$
\begin{aligned}
& x=\frac{\hat{x}}{l}, \quad y=\frac{\hat{y}-\hat{\sigma}(\hat{x})}{l}(G r)^{1 / 4}, \quad r=\frac{\hat{r}}{l}, \quad p=\frac{l^{2}}{\rho v_{\infty}^{2}} G r^{-1} \hat{p}, \quad a=\frac{\hat{a}}{l}, \\
& u=\frac{\rho l}{\mu} G r^{-1 / 2} \hat{u}, \quad v=\frac{\rho l}{\mu} G r^{-1 / 4}\left(\hat{v}-\sigma_{x} \hat{u}\right), \quad \theta=\frac{T-T_{\infty}}{T_{w}-T_{\infty}}, \\
& G r=\frac{g \beta \cos \Omega\left(T_{w}-T_{\infty}\right) l^{3}}{v_{\infty}^{2}}, \quad \sigma(x)=\frac{\hat{\sigma}(\hat{x})}{l}, \sigma_{x}=\frac{d \hat{\sigma}}{d \hat{x}}
\end{aligned}
$$

where $\theta$ is the dimensionless temperature, $\mu$ is the dynamic viscosity and $G r$ is the Grashof number. Substituting the transformations given in (11) into (3)-(6) and ignoring terms of small order in $\mathrm{Gr}$ we obtain the following boundary layer equations:

$$
\begin{aligned}
& \frac{\partial(r u)}{\partial x}+\frac{\partial(r v)}{\partial y}=0 \\
& u \frac{\partial u}{\partial x}+v \frac{\partial u}{\partial y}=-\frac{\partial p}{\partial x}+\sigma_{x} G r^{1 / 4} \frac{\partial p}{\partial y}+\left(1+\sigma_{x}{ }^{2}\right) \frac{\partial^{2} u}{\partial y^{2}}+\theta \\
& \sigma_{x}\left(u \frac{\partial u}{\partial x}+v \frac{\partial u}{\partial y}\right)+\sigma_{x x} u^{2}=-G r^{1 / 4} \frac{\partial p}{\partial y}+\sigma_{x}\left(1+\sigma_{x}^{2}\right) \frac{\partial^{2} u}{\partial y^{2}}+\theta \tan \Omega \\
& u \frac{\partial \theta}{\partial x}+v \frac{\partial \theta}{\partial y}=\frac{1}{\operatorname{Pr}}\left(1+\sigma_{x}{ }^{2}+N_{R}\right) \frac{\partial^{2} \theta}{\partial y^{2}}
\end{aligned}
$$

where $N_{R}=16 \sigma T_{\infty}^{3} / 3 k \alpha^{*}$ is the radiation parameter and $\operatorname{Pr}=\frac{\mu C_{p}}{k}$ is the Prandtl number.

The corresponding boundary conditions to be satisfied are:

$$
\begin{aligned}
& u=0, v=0, \theta=1 \text { at } y=0 \\
& u=0, \theta=0 \text { as } y \rightarrow \infty
\end{aligned}
$$

Equation (13) indicates that the pressure gradient along the $y$-direction is of $O\left(G r^{-1 / 4}\right)$, which implies that lowest order pressure gradient along $x$-direction can be determined from the inviscid flow solution. However, this pressure gradient is zero, since there is no externally induced free stream. On the other hand, equation (14) shows that $G r^{-1 / 4} \partial p / \partial y$ is of $O(1)$ and is determined by the left-hand side of this equation. Thus, the elimination of $\frac{\partial p}{\partial y}$ from equations (13) and (14) leads to

$$
u \frac{\partial u}{\partial x}+v \frac{\partial u}{\partial y}=-\frac{\sigma_{x} \sigma_{x x}}{1+\sigma_{x}{ }^{2}} u^{2}+\left(1+\sigma_{x}{ }^{2}\right) \frac{\partial^{2} u}{\partial y^{2}}+\left(\frac{1-\sigma_{x} \tan \Omega}{1+\sigma_{x}{ }^{2}}\right) \tan \Omega
$$

If we introduce the non similar variables:

$$
\begin{aligned}
& \psi=x^{3 / 4} r f(x, \eta), \quad \eta=x^{-1 / 4} y \\
& \theta=\theta(x, \eta), \quad r=x \sin \Omega
\end{aligned}
$$

where $\eta$ is the pseudo- similarity variable, $f(x, y)$ is dimensionless stream function and $\psi$ is the stream function which is defined according to $u=\partial \psi / \partial y$ and $v=-\partial \psi / \partial x$. Equations (15) and (17) are now transformed to 
$\left(1+\sigma_{x}{ }^{2}\right) f^{\prime \prime \prime}+\left(\frac{7}{4}\right) f f^{\prime \prime}-\left(\frac{1}{2}+\frac{x \sigma_{x} \sigma_{x x}}{1+\sigma_{x}{ }^{2}}\right) f^{\prime 2}+\left(\frac{1-\sigma_{x} \tan \Omega}{1+\sigma_{x}{ }^{2}}\right) \theta=x\left(f^{\prime} \frac{\partial f^{\prime}}{\partial x}-f^{\prime \prime} \frac{\partial f}{\partial x}\right)$

$$
\frac{1}{\operatorname{Pr}}\left(1+\sigma_{x}^{2}+N_{R}\right) \theta^{\prime \prime}+\frac{7}{4} f \theta^{\prime}=x\left(f^{\prime} \frac{\partial \theta}{\partial x}-\theta^{\prime} \frac{\partial f}{\partial x}\right)
$$

The boundary conditions (16) now take the form

$$
\begin{aligned}
& f=f^{\prime}=0, \theta=1 \text { at } \eta=0 \\
& f^{\prime}=0, \theta=0 \text { as } \eta \rightarrow \infty
\end{aligned}
$$

Here the primes denote the differentiation with respect to $\eta$. The equations (19) and (20) subjected to the boundary conditions (21) are solved by using Mathematica program.

The quantities of physical interest are shearing stress and rate of heat transfer in terms of the skin friction coefficient $C_{f}$ and the Nusselt number $N u$, respectively and can be written as

$C_{f}=\frac{\tau_{w}}{\rho U_{\infty}^{2}}$ and $N u=-\frac{\hat{q}_{w} \hat{x}}{k\left(T_{w}-T_{\infty}\right)}$.

Where $\tau_{w}=\mu(n \nabla u)_{\hat{y}=0}$ and $q_{w}=-k(n \nabla T)_{y=0}+\left(n q_{r}\right)_{\hat{y}=0}$, and $n$ is the unit normal to the wavy surface of the cone.

Using the transformation (9) and (16), then $C_{f}$ and $N u$ take the following form:

$$
\begin{aligned}
& \left(\frac{G r}{x}\right)^{1 / 4} C_{f}=\sqrt{1+\sigma_{x}^{2}} f^{\prime \prime}(x, 0) \\
& \left(\frac{G r}{x}\right)^{-1 / 4} N u=-\sqrt{1+\sigma_{x}^{2}}\left(1+N_{R}\right) \theta^{\prime}(x, 0)
\end{aligned}
$$

\section{Results and discussion}

The set of non-linear partial differential equations (19) and (20) satisfying the boundary conditions (21) have been solved numerically using the Mathematica method for several values of the involved parameters, namely Prandtl number $\operatorname{Pr}$, radiation parameter $N_{R}$, wavy surface amplitude parameter $a$ and cone half angle parameter $\Omega$.

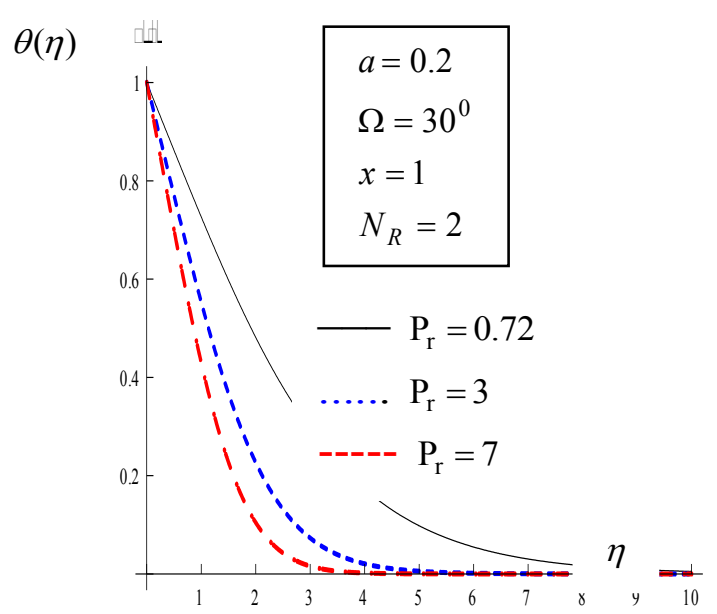

Fig. 2: The temperature profiles $\theta(\eta)$ for

various $P r$ at $a=0.2, \Omega=30^{\circ}, x=1$ and $N_{r}=2$

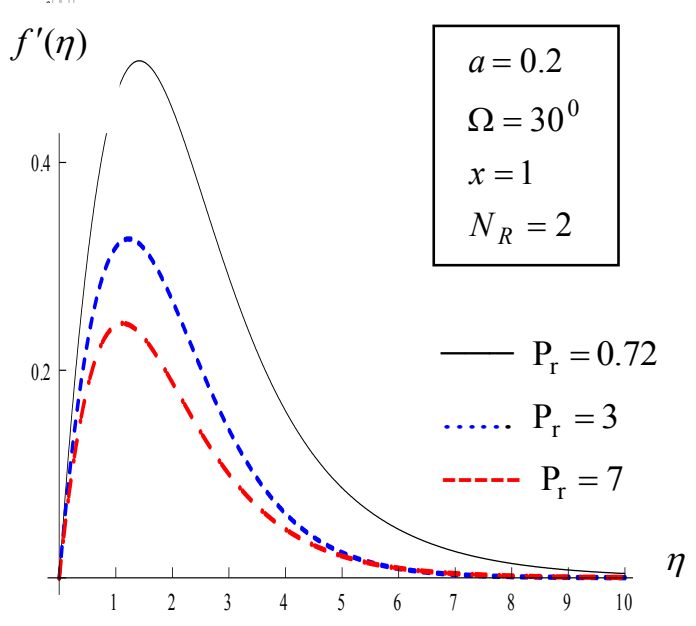

Fig. 3: The velocity profiles $f^{\prime}(\eta)$ for various $\mathrm{Pr}$ at $a=0.2, \Omega=30^{\circ}, x=1$ and $N_{r}=2$ 


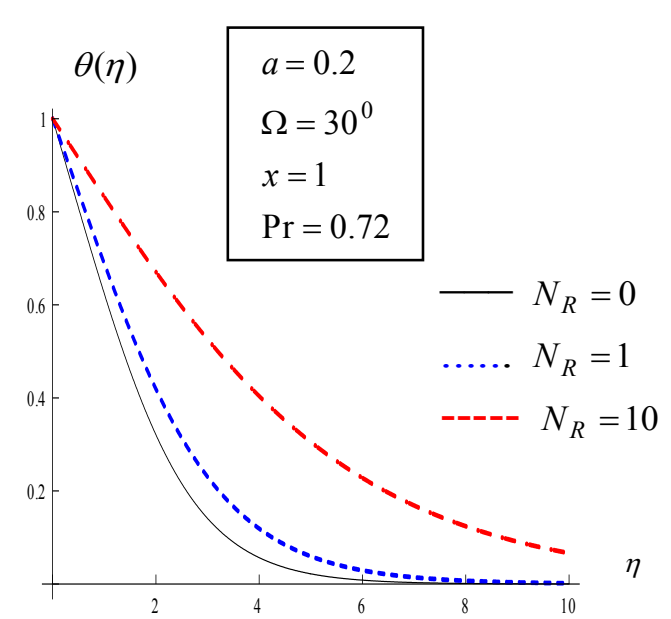

Fig. 5: The temperature profiles $\theta(\eta)$ for various $N_{R}$ at $a=0.2, \Omega=30^{\circ}, x=1$ and $\operatorname{Pr}=0.72$

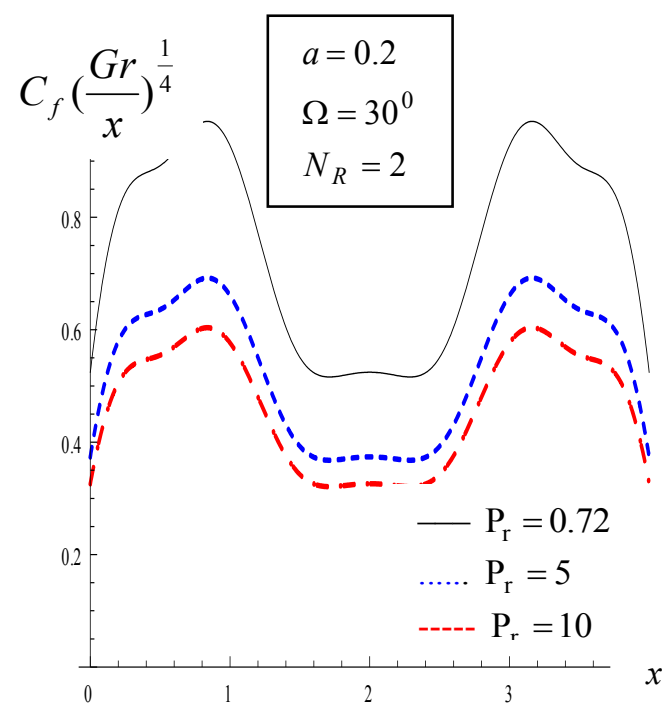

Fig.6: The skin- friction coefficient for various $\operatorname{Pr}$ at $a=0.2, \Omega=30^{\circ}$, and $N_{R}=2$

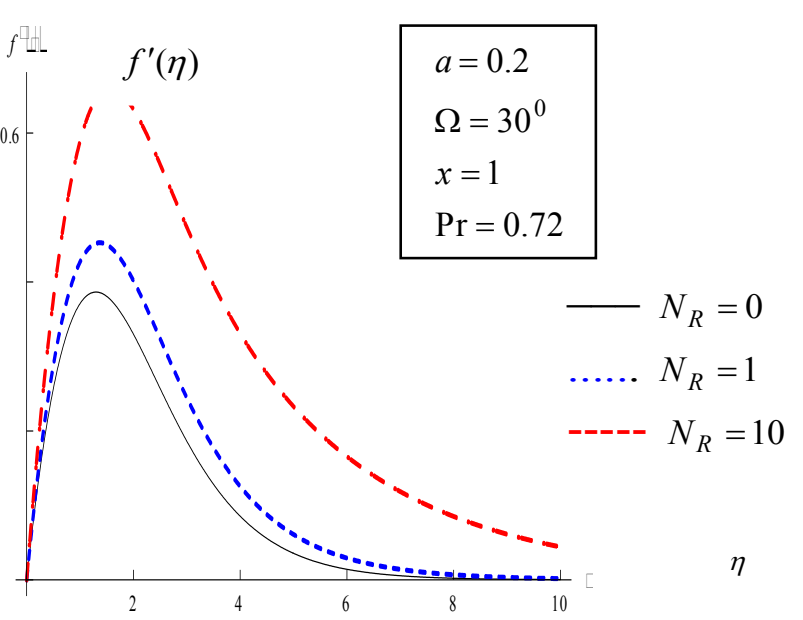

Fig. 4: The velocity profiles $f^{\prime}(\eta)$ for various $N_{R}$ at $a=0.2, \Omega=30^{\circ}, x=1$ and $\operatorname{Pr}=0.72$

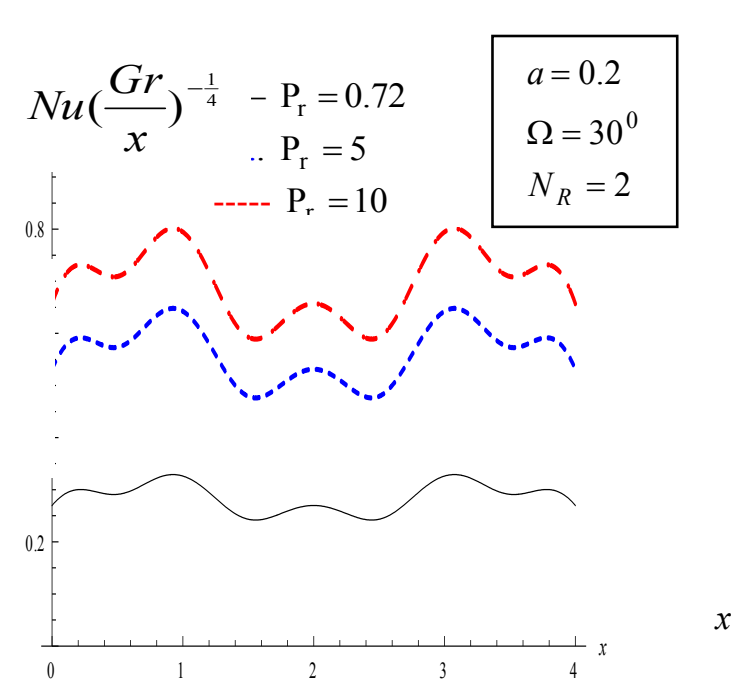

Fig.7: The Nusselt number for various $\operatorname{Pr}$

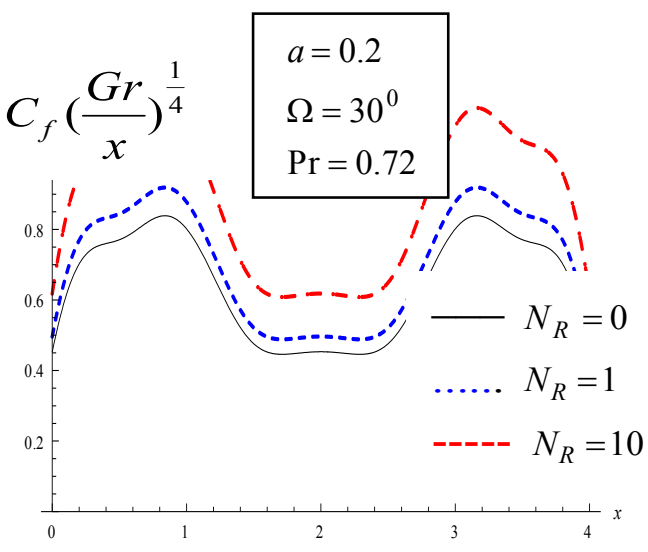

at $a=0.2, \Omega=30^{\circ}$, and $N_{R}=2$

Fig.8: The skin- friction coefficient for various $N_{R}$

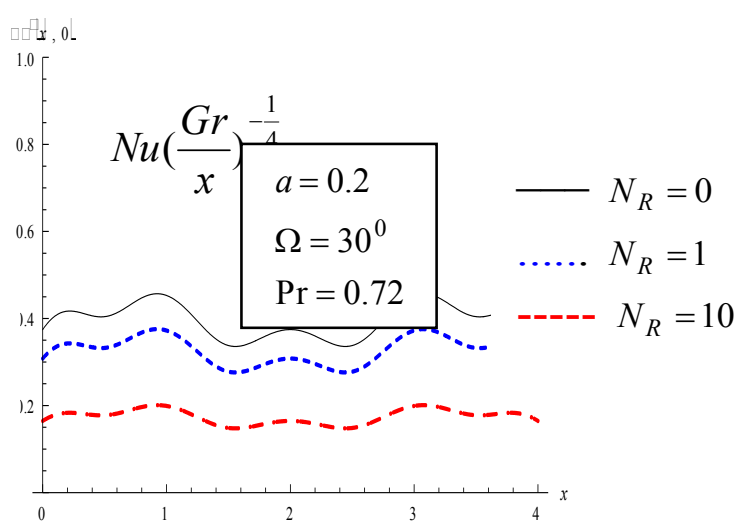

at $a=0.2, \Omega=30^{\circ}$ and $\operatorname{Pr}=0.72$

Fig.9: The Nusselt number for various $N_{R}$ at $a=0.2, \Omega=30^{\circ}$ and $\operatorname{Pr}=0.72$ 
The effects of vary Prandtl $\operatorname{Pr}$ number and radiation parameter $N_{R}$, on the dimensionless velocity profiles $f^{\prime}(\eta)$ and temperature profiles $\theta(\eta)$ are shown in Figures 2-5. Figures $2 \& 3$ show the effects of vary Prandtl number $\operatorname{Pr}$ on the dimensionless velocity and dimensionless temperature against $\eta$ for wavy surface amplitude parameter $a=0.2$ and cone half angle parameter $\Omega=30^{\circ}$, radiation parameter $N_{R}=2$ and the dimensionless distance $x=1$. It can be observed from these figures, the velocity and temperature profiles are decreases for increasing values of Prandtl number $\mathrm{Pr}$. In figures $4 \& 5$ represent the influence of the radiation parameter $N_{R}$, on the velocity and temperature profiles in the boundary layer for $a=0.2, \operatorname{Pr}=0.72$ and $\Omega=30^{\circ}$, while $x=1$. These figures display the effect of radiation parameter, $N_{R}$ on both the velocity and temperature profiles in the boundary layer regime. As radiation parameter, $N_{R}$ increases, both velocity and temperature increase within the boundary layer regime. In figure 4 , for all values of $N_{R}$, there exists a local maximum of the velocity within the boundary layer. As radiation parameter, $N_{R}$ increases, the maximum values of velocity increases. In Figs. 6and 7 found the skin friction coefficient $C_{f}\left(\frac{G r}{x}\right)^{\frac{1}{4}}$ and the rate of heat transfer coefficient $N u\left(\frac{G r}{x}\right)^{-\frac{1}{4}}$ for different values of Prandtl number Pr for $a=0.2, N_{R}=2$ and $\Omega=30^{\circ}$, while $x=1$. The influence of the parameters $N_{R}$ and $a$ for different values of Prandtl number $\mathrm{Pr}$, the decreasing skin friction coefficient becomes slower in the downstream region along $x$ direction. On the other hand, increasing the values of Prandtl number Pr the temperature gradient as well as the rate of heat transfers consequently increases. In Figs. $8 \& 9$, represent the influence of the radiation parameter, $N_{R}$ on the local skin friction coefficient $C_{f}\left(\frac{G r}{x}\right)^{\frac{1}{4}}$ and the rate of heat transfer $N u\left(\frac{G r}{x}\right)^{-\frac{1}{4}}$ for $a=0.2, \operatorname{Pr}=0.72$ and $\Omega=30^{\circ}$, while $x=1$. From Figure 8 , for increasing $N_{R}$, the skin friction coefficient $C_{f}\left(\frac{G r}{x}\right)^{\frac{1}{4}}$ increases. Increasing values of $N_{R}$ leads to increasing the velocity of the fluid (see figure 4) within boundary layer. For enhanced velocity, the velocity gradient of the fluid increases. From figure 9 Increasing values of $N_{R}$ leads to an decrease in the heat absorption intensity of the fluid, consequently the local rate of heat transfer decrease.

\section{IV.Conclusions}

In this paper, the problem of steady laminar free convection flow about a vertical wavy cone in the presence of radiation effect is studied. From the present investigations, we may conclude the following:

1. The velocity and temperature distribution decreases with increasing the values of $\operatorname{Pr}$ and increases with increasing the values of $N_{R}$.

2. For increasing values of the Prandtl number Pr, the skin fiction coefficient decreases but local Nusselt number increases.

3. An increasing in the values of radiation parameter $N_{R}$ leads to increases in the value of the skin friction coefficient while the local heat transfer decrease.

[1] K. C. A. Alam, M. A. Hossain and D. A. S. Rees, Magnetohydrodynamic free convection along a vertical wavy surface, Appl. Mech. Engineering, vol. 1, pp. 555-566, 1997.

[2] I. Pop and T. Y. Na, Natural convection from a wavy cone, Appl. Sci. Research, vol. 54, pp. 125-136, 1995.

[3] I. Pop and T. Y. Na, Natural convection over a vertical wavy frustum of a cone, Int. J. Non-Linear Mechanics, vol. 34, pp. 925-934, 1999.

[4] C. Y. Cheng, Natural convection heat and mass transfer near a vertical wavy cone with constant wall temperature and concentration in a porous medium, Mech. Res. Communication, vol. 27, pp. 613-620, 2000.

[5] M. A. Hossain and D. A. S. Rees, Combined heat and mass transfer in natural convection flow from a vertical wavy surface, Acta Mechanica, vol. 136, pp. 133 141, 1999. 
[6] M. A. Hossain, M. S. Munir and I. Pop, Natural convection flow of viscous fluid with viscosity inversely proportional to linear function of temperature from a vertical cone, Int. J. Therm. Science, vol. 40, pp. 366-371, 2001.

[7] M. A. Hossain, S. Kabir and D. A. S. Rees, Natural convection of fluid with temperature dependent viscosity from heated vertical wavy surface, ZAMP, vol. 53, pp. 48-52, 2002.

[8] T. Cebeci and P. Bradshaw, Physical and Computational Aspects of Convective heat Transfer, Springer, New York, 1984.

[9] C. C. Wang and C. K. Chen, Mixed convection boundary layer flow on inclined wavy plates including the magnetic field effect, Int. J. Therm. Science, vol. 44, pp. 577-586, 2005.

[10] L. S. Yao, Natural convection along a vertical complex wavy surface, Int. J. Heat Mass Transfer, vol. 49, pp.281-286, 2006.

[11] M. M. Molla, M. A. Hossain and L. S. Yao, Natural convection flow along a vertical complex wavy surface with uniform heat flux, ASME J. Heat Transfer, vol. 129 (no.10), pp. 1403-1407, 2007.

[12] Azad Rahman, Md M. Molla, M.M.A. Saker and A. Thohura, Effects of temperature dependent viscosity on natural convection flow along a vertical wavy cone with heat flux, Int. J. of Energy and Technology, 3(8), pp.1-10, 2011.

[13] Hossain M.A., Alim M.A. and Rees D., The effect of radiation on free convection from a porous vertical plate. Int. J. Heat Mass Transfer, 42, pp.181-191, 1999.

[14] Hossain M.A., Khanafer K. and Vafai K., The effect of radiation on free convection flow of fluid with variable viscosity from a porous vertical plate. Int. J. Therm. Sci., 40, pp.115-124, 2001.

[15] Raptis A., Perdikis C. and Takhar H.S., Effect of thermal radiation on MHD flow, Appl. Math. Comp., 153, pp.645-649, 2004.

[16] Cortell R., Effects of heat source/sink, radiation and work done by deformation on flow and heat transfer of a viscoelastic fluid over a stretching sheet. Comput. Math. Appl., 53pp. 305-316, 2007.

[17] Cortell R., Similarity solutions for boundary layer flow and heat transfer of a FENE-P fluid with thermal radiation. Phys. Lett. A., 372, pp.2431-2439, 2008.

[18] Cortell R., Effects of viscous dissipation and radiation on the thermal boundary layer over a nonlinearly stretching sheet. Phys. Lett. A., 372, pp.631-636, 2008.

[19] Cortell R., Radiation effects in the Blasius flow, Appl. Math. Comp., 198, pp. 333-338, 2008. 